\title{
Multiplicity of periodic solutions in bistable equations
}

\author{
Gregory Berkolaikoł and Michael Grinfeld \\ Department of Mathematics, \\ University of Strathclyde, \\ Livingstone Tower, 26 Richmond Street, \\ Glasgow G1 1XH, Scotland, U.K. \\ E-mail: Gregory.Berkolaiko@math.tamu.edu
}

\begin{abstract}
We study the number of periodic solutions in two first order nonautonomous differential equations both of which have been used to describe, among other things, the mean magnetization of an Ising magnet in the time-varying external magnetic field. When the strength of the external field is varied, the set of periodic solutions undergoes a bifurcation in both equations. We prove that despite profound similarities between the equations, the character of the bifurcation can be very different. This results in a different number of coexisting stable periodic solutions in the vicinity of the bifurcation. As a consequence, in one of the models, the Suzuki-Kubo equation, one can effect a discontinuous change in magnetization by adiabatically varying the strength of the magnetic field.

PACS number: 05.50.+q
\end{abstract}

\section{Introduction}

In many lattice models the mean field approximation leads to ODEs with periodically time dependent right-hand side. For example, the Curie-Weiss model with Glauber spin dynamics in the thermodynamic limit leads to the so-called Suzuki-Kubo equation (see the derivation in [3, Ch. 3] or in [11, 12, and a numerical study in [1]).

The Suzuki-Kubo equation is

$$
\epsilon \frac{d m}{d \tau}=-m+\tanh [\beta(m+\tilde{h} \cos (2 \pi \tau))],
$$

where $m$ is the average magnetization of a sample, $\epsilon$ is the relaxation time, $\tilde{h}$ is the amplitude of the applied magnetic field, and $\beta$ is $1 / k T$, with $T$ the absolute temperature.

A seemingly similar first order non-autonomous equation,

$$
\epsilon \frac{d x}{d t}=a x+b x^{3}+h \cos (2 \pi t), a, b \in \mathbb{R},
$$

$\ddagger$ Present address: Mathematics, Texas A\&M University, College Station, TX 77843-3368, USA 
has been used as a simple generic model for switchable bistable systems. If $a>0$ and $b<0$, it describes the overdamped dynamics of a particle in a quadratic potential with periodic forcing. Such equations have been studied in the context of laser optics (longitudinal mode instabilities in a semiconductor laser); see [7, 8] for analysis and references to the optics literature.

The issue we want to address is the number of $2 \pi$-periodic solutions for various values of $h$ and other parameters. In particular, we will use $h$ as the bifurcation parameter. This issue is of physical importance, since one wants to determine whether it is possible, say, in the Suzuki-Kubo context, to effect a discontinuous change in magnetization by continuously varying the amplitude of the applied magnetic field $h$ (i.e. a first order phase transition).

To compare the two equations effectively, we rescale the variables in (11) in the following way: set, in sequence,

$$
x=m+\tilde{h} \cos (2 \pi \tau), \quad h=\tilde{h} \sqrt{1+4 \pi^{2} \epsilon^{2}}, \quad t=\tau+\frac{1}{2 \pi} \arccos \left(\frac{1}{\sqrt{1+4 \pi^{2} \epsilon^{2}}}\right) .
$$

Then in the new variables we have

$$
\epsilon \frac{d x}{d t}=-x+\tanh \beta x+h \cos (2 \pi t) .
$$

Comparing the two equations, we see that the nonlinearity in (44) is sublinear while it is superlinear in (2). Furthermore, $\tanh (z)$ is not an entire function. Note also that the Taylor series expansion of $-x+\tanh \beta x$ is

$$
-x+\tanh \beta x=(\beta-1) x-\frac{\beta^{3}}{3} x^{3}+O\left(x^{5}\right) .
$$

Hence superficially it looks that the behaviour of solutions of (41) should be similar to that of (2) with $a=\beta-1$ and $b=-\beta^{3}$. In fact, this is the implicit assumption under much of the physical literature. This assumption is incorrect. It turns out that for certain values of $\beta$ and $h$ equation (4) has 5 periodic solution, a situation which is impossible for equation (2). The numerical simulations confirming this were reported in, for example, [3, Ch. 7]. Here we aim to establish this fact analytically.

The structure of the paper is as follows. After collecting preliminaries below, in section 3 we perform the Liapunov-Schmidt reduction of the general equation,

$$
\frac{d x}{d t}=f(x)+h \cos (2 \pi t)
$$

The reduction is complicated by the fact that we do not know the bifurcating solution explicitly.

In section 4 we use the results of the reduction to conclude that for small $\beta$ behaviour of (44) is similar to that of (2). Then we consider the case of large $\beta$ in (4) by explicitly examining the case of $\beta=\infty$. The limiting equation is in fact a differential inclusion which we analyse explicitly, finding the range of $h$ for which 5 solutions coexist. Appealing to a continuation theorem [2], we conclude that such behaviour persist for large, but finite, values of $\beta$ as well. 


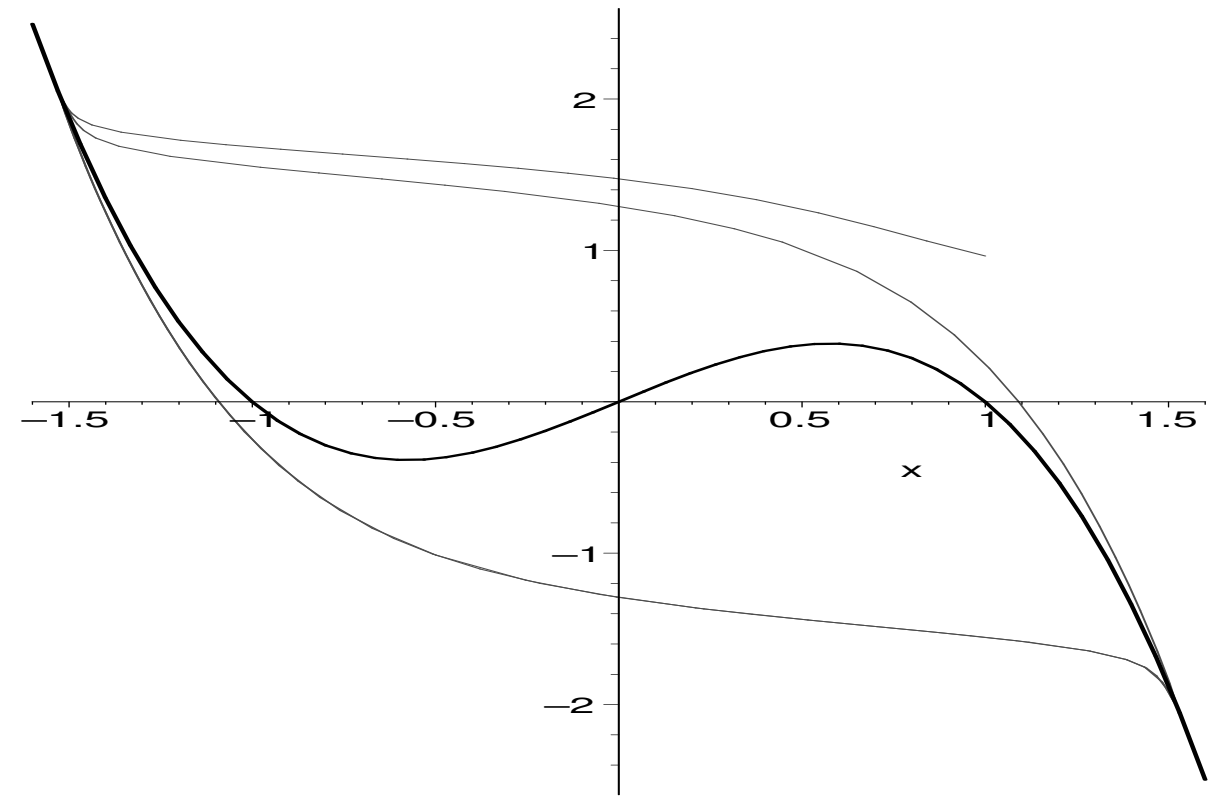

Figure 1. The picture of the (stable) hysteresis loop for large $h$. In this case we take $f(x)=x-x^{3}, \epsilon=0.05$ and $h=2.0$.

Everywhere below we assume that the function $f(\cdot)$ is sufficiently smooth. As can be seen from ([6), we fix $\epsilon=1$, as this does not affect any of the results. However in our numerics we take $\epsilon$ of the order of 0.05 which "slows down" the time and results in nicer plots.

Whenever we sketch the solutions of the equation we do it in $(x, h \cos (2 \pi t))$ plane, i.e. we plot the curve $(x(t), y(t))$ where

$$
\left\{\begin{array}{l}
\dot{x}=f(x)-y, \\
y=h \cos (2 \pi t) .
\end{array}\right.
$$

This makes it easier to identify the periodic solutions (they are closed curves) and produces familiar hysteresis loop pictures for large $h$, figure [

\section{Background information}

The examples of function $f(x)$ that we are going to consider here, $f(x)=\tanh \beta x-x$, $\beta>1$ and $f(x)=\gamma x-x^{3}, \gamma>0$ share many important properties. Both are odd functions and the equation $f(x)=0$ has exactly three solutions. If we consider equation (6) for $h=0$, there will correspondingly be three stationary solutions. The central stationary solution $x(t)=0$ will be unstable and the other two are locally asymptotically stable.

It follows from the implicit function theorem that for small $h$, there will be three periodic solutions, two stable and one unstable. Below we show that this situation persists at least until $h_{0}=\max _{x>0} f(x)$. Also we will show that for large $h$ there is only one, stable, solution. The main aim of this paper is to uncover the bifurcation picture 


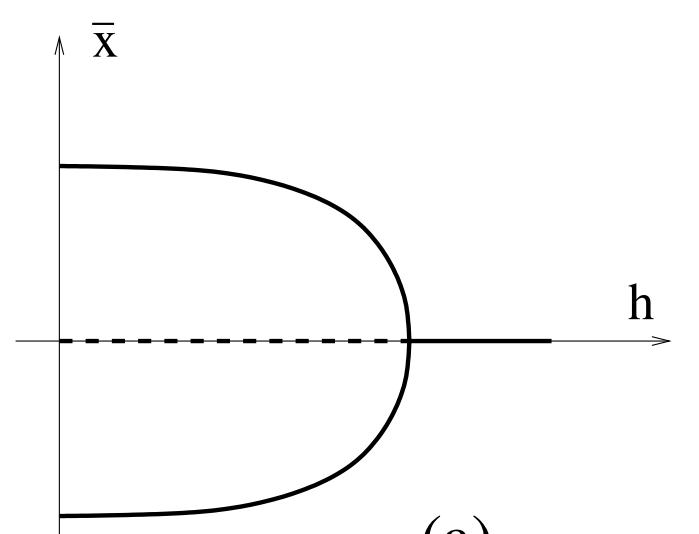

(a)

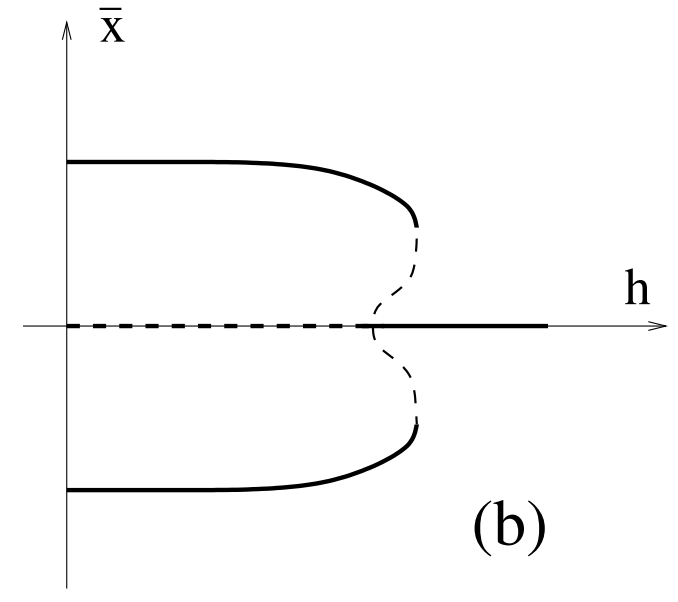

Figure 2. Two possible minimal scenarios of the emergence of a single periodic solution. In scenario (a) three solutions merge in a subcritical pitchfork bifurcation. In scenario (b) the central solution first undergoes a supercritical pitchfork bifurcation, emitting two unstable solutions. These unstable solutions disappear in fold bifurcations upon meeting the stable solutions.

for the intermediate values of $h$. The simplest scenario where three solutions can merge into one is a subcritical pitchfork as shown on figure 2(a). There, for simplicity, we sketch the means of periodic solutions, $\bar{x}=\int_{0}^{1} x(t) d t$, versus $h$.

Contrary to the above expectations, numerical investigations of the Suzuki-Kubo equation (44) performed in [13] (see also 3, Ch. 3] and [2]) show that for large values of $\beta$ and the parameter $h$, the equation has three stable periodic solutions (and therefore five periodic solutions altogether). The bifurcation diagram would then look like figure 2(b). For small values of $\beta$, 13] reported two stable periodic solutions at most, a situation compatible with figure 2(a). The critical value of $\beta$ at which the bifurcation picture changed was referred to as the tricritical points (TCP).

The existence of TCP was disputed by [14] (see also 9]), who studied equation (2) numerically and reported that, although the stable non-symmetric solution changed fast in the vicinity of bifurcation, it was not disappearing in a "blue-sky catastrophe" as in figure 2(b).

Our investigation concludes that, from mathematical point of view, both results are correct: there are at most 3 solutions in (2) and there is a TCP in equation (44).

\subsection{Periodic solutions for small $h$}

Theorem 2.1. Let $f(x)$ be a continuously differentiable function satisfying

$$
f^{\prime}(x)<0 \quad x \in(a, b) \text { and } f(a)>0>f(b) .
$$

Put $h_{0}=\min (f(a),-f(b))$. Then for any $h<h_{0}$ there is a unique periodic solution to equation (6) satisfying $x(t) \in[a, b]$ for all $t$. This solution is stable.

Proof. The idea of the proof is to construct such a trapping region that any solution that enters it must stay there. Then we can appeal to a fixed point theorem to infer 
that there is a periodic solution inside this region. Analysing stability of this solution we find that it must be stable, therefore it is unique.

The function $f(x)$ is monotone therefore there are unique solutions to the equations $f\left(x_{a}\right)=h_{0}$ and $f\left(x_{b}\right)=-h_{0}$. Since, for any $t, x(t)=x_{a}$ implies $x^{\prime}(t)=f\left(x_{a}\right)+$ $h \cos (2 \pi t) \geq h_{0}-h>0$, any solution to the right of $x_{a}$ will remain there. Similarly, solutions to the left of $x_{b}$ will never increase past $x_{b}$. Thus $\left[x_{a}, x_{b}\right]$ is a trapping region and there is at least one periodic solution.

Linearizing equation (6) shows that a solution $x_{0}(t)$ is stable if

$$
\lambda=\int_{0}^{1} \frac{\partial f}{\partial x}(\hat{x}(s)) d s
$$

is negative. But since $f^{\prime}(x)<0$ for all $x \in\left[x_{a}, x_{b}\right]$, every periodic solution is stable.

Corollary 2.2. Let $x_{m}$ be such that $f\left(x_{m}\right)=\max _{x>0} f(x)$, where $f(x)$ is either $\tanh \beta x-x$ or $\gamma x-x^{3}$. Then for $h<f\left(x_{m}\right)$ equation (6) has exactly three solutions, one in each of the intervals $\left(-\infty,-x_{m}\right),\left(-x_{m}, x_{m}\right)$ and $\left(x_{m}, \infty\right)$. Two of these solutions are stable and one is unstable.

\subsection{Uniqueness of periodic solution for large $h$}

For large $h$ we have the following general theorem, which, in particular, is valid for both functions $f(x)$ that are of interest to us.

Theorem 2.3. Let $f(x)$ be a continuous almost everywhere differentiable function. If its derivative satisfies

$$
f^{\prime}(x)<\alpha<0 \quad \text { for }|x|>x_{0} \quad \text { and } \quad f^{\prime}(x)<\beta \quad \text { for all } x
$$

for some $\alpha, \beta$ and $x_{0}$, then, for sufficiently large $h$, equation (6) has a unique stable periodic solution.

Proof. Existence. Property (10) implies that $f(x) \rightarrow \mp \infty$ eventually monotonically as $x \rightarrow \pm \infty$. This means, in particular, that the equations $f(x)=h$ and $f(x)=-h$ have exactly one solution each for sufficiently large $h$. Denoting these solutions by $x_{h-}$ and $x_{h+}$, we notice that the interval $\left[x_{h-}, x_{h+}\right]$ is trapping for the trajectories of (10). Hence there must be at least one periodic solution.

Uniqueness. We will prove the stability of all periodic solutions which will imply that there can be at most one. Let $\hat{x}(t)$ be a periodic solution, which is stable if $\lambda$, defined by equation (9), is negative. Denoting the time spent by the solution inside $\left[-x_{0}, x_{0}\right]$ by $t_{+}$, we can estimate $\lambda$ by

$$
\lambda<\beta t_{+}+\alpha\left(1-t_{+}\right) .
$$

We aim to show that the time $t_{+} \rightarrow 0$ as $h \rightarrow \infty$ and hence $\lambda<0$.

To do it we define three regions in the plane $(x, h \cos (2 \pi t))$ which add up to the strip $|x|<x_{0}$, see figure 3 . The region $A$ is defined by $|h \cos (2 \pi t)| \leq y_{0}$, the region $B$ 


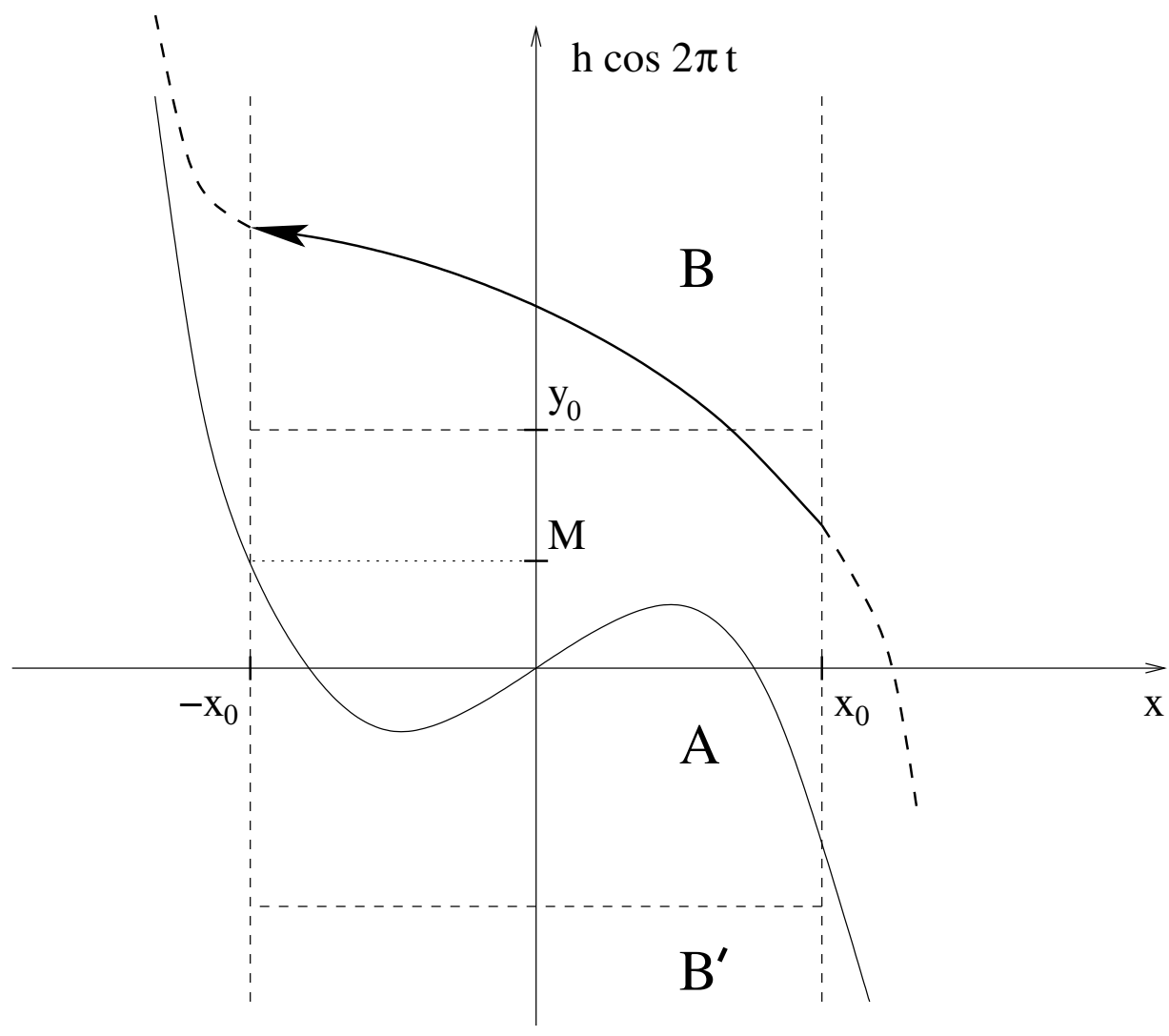

Figure 3. Sketch of an orbit crossing $\left[-x_{0}, x_{0}\right]$ and the three regions dividing the strip.

by $h \cos (2 \pi t)>y_{0}$ and the region $B^{\prime}$ by $h \cos (2 \pi t)<-y_{0}$. For now, we leave the choice of $y_{0}$ open.

From the definition of region $A$, the time spent by the solution in $A$ is at most

$$
t_{A}=2 \frac{1}{2 \pi} \arcsin \left(\frac{y_{0}}{h}\right) .
$$

If $M$ is the maximum of $|f(x)|$ on $\left[-x_{0}, x_{0}\right]$, then $\left|x^{\prime}(t)\right|>y_{0}-M$ and the time spent by the solution in $B$ or $B^{\prime}$ during one visit is at most

$$
t_{B}=\frac{2 x_{0}}{y_{0}-M}
$$

It is easy to see that a periodic solution can visit $B$ at most once during one period (and at most twice if it is not periodic); the same applies to region $B^{\prime}$. Thus we conclude that the total time spent in the strip $|x|<x_{0}$ is

$$
t_{+}<\frac{1}{\pi} \arcsin \left(\frac{y_{0}}{h}\right)+\frac{4 x_{0}}{y_{0}-M} .
$$

Now if we take $y_{0}$ to be equal, for example, to $\sqrt{h}$, the right hand side will tend to zero as $h \rightarrow \infty$. 


\section{The Liapunov-Schmidt reduction}

In this section we assume that function $f(y)$ in equation (6) is odd. It immediately follows that if $x(t)$ is a solution of (6) , then so is $\widetilde{x}(t)=-x(t+1 / 2)$. In particular, there is always a "symmetric solution" which satisfies $x(t)=-x(t+1 / 2)$.

We shall perform the Liapunov Schmidt reduction (LSR) for equation (6) aiming to determine the character of the bifurcation of the symmetric solution as we change the parameter $h$. We shall denote by $h_{c s}$ the corresponding $\oint$ critical value of $h$.

We start by defining the operator $\Phi$ by

$$
\Phi(x)=\dot{x}-f(x)-h \cos (2 \pi t) .
$$

The periodic orbits are the zeros of this operator in the space of continuously differentiable 1 -periodic functions $C_{p}^{1}([0,1])$ contained in $L^{2}([0,1])$. The reduction, based on a particular solution $x_{0}(t)$, leads to the construction of a reduced function $g: \mathbb{R} \times \mathbb{R} \rightarrow \mathbb{R}$ such that the solutions of $g(x, h)=0$ are locally in one-to-one correspondence with the solutions of (15), with the solution $x_{0}(t)$ corresponding to the zero solution of $g(x, h)=0$. It is rarely possible to compute $g(x, h)$ explicitly, but one can examine the bifurcation picture by computing the derivatives of $g$.

First of all, however, we describe the symmetric solution $x_{0}(t)$ at the critical point $h=h_{c s}$.

\subsection{The bifurcation condition}

The necessary bifurcation condition is that the differential of $\Phi$ has non-zero kernel. This is because if $\operatorname{Ker}\left(d \Phi\left(x_{0}\right)\right)=\{0\}$, the solution $x_{0}$ of the equation $\Phi\left(x_{0}, h_{c s}\right)=0$ can be uniquely continued by the implicit function theorem. In our case the differential of $\Phi$, which we denote by $L$, is

$$
d \Phi(x)\left(v_{1}\right)=\left.\frac{d}{d \xi} \Phi\left(x+\xi v_{1}\right)\right|_{\xi=0}=\dot{v}_{1}-\frac{\partial f}{\partial x}(x) v_{1} \equiv L v_{1}
$$

Therefore, the necessary condition for bifurcation at $x_{0} \equiv x_{0}(h, t)$ is that there is a non-zero 1-periodic solution to

$$
\dot{v}=\frac{\partial f}{\partial x}\left(x_{0}\right) v .
$$

The general solution of (17) is

$$
v(t)=C \exp \left(\int_{0}^{t} \frac{\partial f}{\partial x}\left(x_{0}(s)\right) d s\right) .
$$

This solution is 1-periodic iff

$$
\int_{0}^{1} \frac{\partial f}{\partial x}\left(x_{0}(s)\right) d s=0
$$

which we will refer to as the bifurcation condition.

$\S$ the subscripts "cs" stand for "critical symmetric" 
To proceed with the LSR we need to find a basis for $\operatorname{Ker} L$ and (Range $L)^{\perp}$. The former is spanned by the function $v(t)$ defined by (18) with, for example, $C=1$. The latter satisfies

$$
0=\left\langle v^{*}, L y\right\rangle=\left\langle-\frac{d}{d t} v^{*}-\frac{\partial f}{\partial x}\left(x_{0}(t)\right) v^{*}, y\right\rangle
$$

for all $y$. Solving equation

$$
\dot{v}^{*}=-\frac{\partial f}{\partial x}\left(x_{0}\right) v^{*}
$$

and again setting the arbitrary constant equal to one, we obtain

$$
v^{*}(t)=\exp \left(-\int_{0}^{t} \frac{\partial f}{\partial x}\left(x_{0}(s)\right) d s\right) .
$$

Note that with our choice of arbitrary constants $v(t) v^{*}(t) \equiv 1$ which will be used often in what follows.

\subsection{Symmetries of the reduced function}

The function $g(x, h)$ inherits the symmetries of the original operator $\Phi(x)$. In our case, the following proposition from [6, 4.3] holds

Proposition 1. Let $\operatorname{dim} \operatorname{Ker} L=\operatorname{dim} \operatorname{Range} L=1$ and let $\operatorname{Ker} L=\operatorname{span}\{v\}$. If there is a symmetry operator $R: C_{p}^{1}([0,1]) \rightarrow C_{p}^{1}([0,1])$ which satisfies

$$
R^{2} x=x, \quad \Phi(R x)=R \Phi(x) \quad \text { and } \quad R x_{0}(t)=x_{0}(t),
$$

for all $x \in C_{p}^{1}([0,1])$ then $R v(t)$ is equal to either $v(t)$ or $-v(t)$. In the latter case the reduced function is odd in $x: g(-x, h)=-g(x, h)$.

It is easy to check that the symmetry operator satisfying conditions (23) is $R \phi(t)=-\phi(t+1 / 2)$. Since $v(t)$ is an exponential (see (18) ), its sign does not change

over $[0,1]$ and $R v(t)=-v(t+1 / 2)$ must be equal to $-v(t)$. Therefore our reduced function is indeed odd.

As a consequence, we immediately get

$$
g(0, h)=0, \quad g_{x x}(0, h)=0, \quad \text { etc. }
$$

In addition, $g_{x}\left(0, h_{c s}\right)=0$ since $h_{c s}$ is critical. Thus, to investigate the pitchfork bifurcation of the symmetric solution of (6) , if the bifurcation is indeed a pitchfork, it suffices to study $g_{x h}\left(0, h_{c s}\right)$ and $g_{x x x}\left(0, h_{c s}\right)$.

\subsection{Bifurcation function}

To study the remaining derivatives of $g(x, h)$ we compute higher derivatives of the operator $\Phi$ (here $\left.v_{1}, v_{2}, v_{3} \in C_{p}^{1}([0,1])\right)$ :

$$
\begin{aligned}
& d^{2} \Phi(x)\left(v_{1}, v_{2}\right)=-\frac{\partial^{2} f}{\partial x^{2}}(x) v_{1} v_{2} \\
& d^{3} \Phi(x)\left(v_{1}, v_{2}, v_{3}\right)=-\frac{\partial^{3} f}{\partial x^{3}}(x) v_{1} v_{2} v_{3}
\end{aligned}
$$


Multiplicity of periodic solutions in bistable equations

$$
\begin{array}{ll}
\frac{\partial}{\partial h} \Phi & =-\cos (2 \pi t) \\
d\left(\frac{\partial}{\partial h} \Phi\right) & =0 .
\end{array}
$$

We have already concluded that

$$
\begin{aligned}
& g_{h}=\left\langle v^{*}, \frac{\partial}{\partial h} \Phi\right\rangle=0, \\
& g_{x x}=\left\langle v^{*}, d^{2} \Phi(v, v)\right\rangle=0 .
\end{aligned}
$$

These formulae will come in handy below.

The derivative $g_{h x}$ is given by

$$
g_{h x}=\left\langle v^{*}, d\left(\frac{\partial}{\partial h} \Phi\right) v-d^{2} \Phi\left(v, L^{-1} E\left(\frac{\partial}{\partial h} \Phi\right)\right)\right\rangle,
$$

where $E$ is the projection to Range $L$,

$$
E y=y-\left\langle v^{*}, y\right\rangle \frac{v^{*}}{\left\|v^{*}\right\|} .
$$

In our case we have $d\left(\frac{\partial}{\partial h} \Phi\right)=0$ and, since $\left\langle v^{*}, \frac{\partial}{\partial h} \Phi\right\rangle=g_{h}=0$, the projector $E$ leaves $\frac{\partial}{\partial h} \Phi$ invariant. Taking into account that $v^{*} v \equiv 1$ we get

$$
g_{h x}=-\left\langle v^{*}, d^{2} \Phi\left(v, L^{-1}\left(\frac{\partial}{\partial h} \Phi\right)\right)\right\rangle=\int_{0}^{1} \frac{\partial^{2} f}{\partial x^{2}}\left(x_{0}(s)\right) u(s) d s,
$$

where $u(s)=L^{-1}\left(\frac{\partial}{\partial h} \Phi\right)$ is the solution of

$$
\dot{u}-\frac{\partial f}{\partial x}\left(x_{0}(s)\right) u=-\cos (2 \pi t) \text {. }
$$

Differentiating the identity $\Phi\left(x_{0}(h, t)\right)=0$ with respect to $h$, we notice that $u(s)=$ $-\partial x_{0}(h, s) / \partial h$. Eventually we get

$$
g_{h x}\left(0, h_{c s}\right)=-\frac{\partial}{\partial h} \int_{0}^{1} \frac{\partial f}{\partial x}\left(x_{0}(s)\right) d s
$$

therefore, at the stability-gaining bifurcation we should have

$$
g_{h x} \geq 0 .
$$

Remark 1. One consequence of theorem 2.3 is that the symmetric solution has to undergo at least one bifurcation in which it turns from being unstable to being stable. As of now, we are unable to prove that it undergoes exactly one bifurcation which is equivalent to showing that $g_{h x}$ is strictly greater than zero at all bifurcations.

The character of the stability-gaining bifurcation is determined by the sign of $g_{x x x}$,

Theorem 3.1. If $g_{x x x}\left(0, h_{c s}\right)$ is positive (corresp. negative), the stability-gaining bifurcation from the zero-mean solution is a subcritical (corresp. supercritical) pitchfork. 
Proof. We expand $g(x, h)$ in Maclaurin series in $x$, taking into account that the function is odd,

$$
g(x, h)=g_{x}(0, h) x+g_{x x x}(0, h) \frac{x^{3}}{6}+O\left(x^{5}\right)
$$

To understand the bifurcation picture, it suffices to take into account only the first two terms. If $g_{x x x}(0, h)>0$, the equation

$$
g_{x}(0, h) x+g_{x x x}(0, h) \frac{x^{3}}{6}=0
$$

has 3 solutions if $g_{x}(0, h)<0$ and 1 solution otherwise. Since $g_{x x x}\left(0, h_{c s}\right)>0$ it remains so in a small neighbourhood of $h=h_{c s}$. Thus the main question is the sign of $g_{x}(0, h)$.

To prove that, as $h$ crosses $h_{c s}, g_{x}(0, h)$ goes from being negative to being positive, we can show that $\|$

$$
g_{x}(0, h)=-\int_{0}^{1} \frac{\partial f}{\partial x}\left(x_{0}(s)\right) v(s) d s=-\lambda
$$

which is rather involved. Alternatively we can quote a result connecting the sign of $g_{x}(x, h)$ with the stability of the corresponding solution of the original differential equation [6]. In our case, the solution corresponding to $(0, h)$ is the symmetric solution which is stable (unstable) whenever $g_{x}(0, h)$ is positive (corresp. negative). Since the bifurcation is stability-gaining, we get three solutions for $h<h_{c s}$ and one solution for $h>h_{c s}$.

To study the sign of $g_{x x x}$ we use the following formula

$$
g_{x x x}=\left\langle v^{*}, d^{3} \Phi(v, v, v)-3 d^{2} \Phi\left(v, L^{-1} E d^{2} \Phi(v, v)\right)\right\rangle
$$

From the definition of the projector $E$, see (32), and identity (30) we conclude that $E d^{2} \Phi(v, v)=d^{2} \Phi(v, v)$. Further, inverting $L$ we get

$L^{-1} d^{2} \Phi(v, v)=v(t) \int_{0}^{t} \Phi(v, v) v^{*}(s) d s=-v(t) \int_{0}^{t} \frac{\partial^{2} f}{\partial x^{2}}(x(s)) v(s) d s$.

First we evaluate the second part of (40),

$$
\begin{aligned}
-3\left\langle v^{*}, d^{2} \Phi\right. & \left.\left(v, L^{-1} E d^{2} \Phi(v, v)\right)\right\rangle \\
& =-3 \int_{0}^{1} v^{*}(t) \frac{\partial^{2} f}{\partial x^{2}}(x) v^{2}(t)\left(\int_{0}^{t} \frac{\partial^{2} f}{\partial x^{2}}(x(s)) v(s) d s\right) d t
\end{aligned}
$$

which can be written as

$$
-3 \int_{0}^{1} w^{\prime}(t) w(t) d t=\left[-\frac{3 w^{2}(t)}{2}\right]_{0}^{1}=0
$$

where $w(t)=\int_{0}^{t} \frac{\partial^{2} f}{\partial x^{2}}(x(s)) v(s) d s$. The integral above is equal to zero since $w(1)=g_{x x}=$ 0 . 
Thus, in our case,

$$
g_{x x x}=\left\langle v^{*}, d^{3} \Phi(v, v, v)\right\rangle=-\int_{0}^{1} \frac{\partial^{3} f}{\partial x^{3}}\left(x_{0}(s)\right) v^{2}(s) d s .
$$

Corollary 3.2. The stability-gaining bifurcation of the zero-mean solution of (6) with $f(x)=x-x^{3}$ is a subcritical pitchfork.

Proof. In this case $\frac{\partial^{3} f}{\partial x^{3}} \equiv-6$ and, since $v^{2}$ is non-zero, $g_{x x x}>0$.

A different proof of this (local) theorem for cubic $f$ is given by Byatt-Smith [5]. That in the optical bistability equation (2) one can never have more than three 1-periodic solutions is proved very elegantly in [10].

The above results will be used in section 4.1 to show that locally the situation in the Suzuki-Kubo equation (4) is similar to (2) if $\beta$ is sufficiently small.

\section{The Suzuki-Kubo equation}

In this section we study the case

$$
f(x)=x-\tanh (\beta x)
$$

in detail.

We aim to demonstrate that for sufficiently large $\beta$ there are values of $h$ for which there exist at least 5 periodic solutions of (44). We also show that for small $\beta$ the bifurcation of the zero-mean solution is a subcritical pitchfork (figure 2(a)) which means that the maximum number of solutions is 3. The boundary between these two regimes is known as the tricritical point (TCP).

\subsection{The case of small $\beta$}

Theorem 4.1. There exists $\beta_{0}$ such that for all $1<\beta<\beta_{0}$ and the zero-mean periodic solution $x_{0}(t)$ satisfying (19) we have

$$
\int_{0}^{1} \frac{\partial^{3} f}{\partial x^{3}}\left(x_{0}(s)\right) v^{2}(s) d s<0 .
$$

It is not hard to get the rough estimate, $\beta_{0}=4 / 3$, which is sufficient for our purposes.

Proof. Put $\tanh \left(\beta x_{0}(s)\right)=\zeta(s)$. From (17), we have

$$
\int_{0}^{1} \frac{\partial f}{\partial x}\left(x_{0}(s)\right) v^{n}(s) d s=\int_{0}^{1} v^{n-1}(s) \dot{v}(s) d s=0 \quad \text { for all } n \geq 1,
$$

since $v(t)$ is 1-periodic. Differentiating (45),

$$
\frac{\partial f}{\partial x}\left(x_{0}(s)\right)=\beta-1-\beta \zeta^{2}(s),
$$


multiplying it by $v^{2}(s)$ and choosing $n=2$ in (47), we have

$$
\int_{0}^{1} \zeta^{2}(s) v^{2}(s) d s=\frac{\beta-1}{\beta} \int_{0}^{1} v^{2}(s) d s .
$$

For the third derivative of $f(x)$ we now get

$$
\begin{aligned}
\int_{0}^{1} \frac{\partial^{3} f}{\partial x^{3}}\left(x_{0}(s)\right) & v^{2}(s) d s \\
& =-2 \beta^{3} \int_{0}^{1} v^{2}(s) d s+8 \beta^{3} \int_{0}^{1} v^{2}(s) \zeta^{2}(s) d s-6 \beta^{3} \int_{0}^{1} \zeta^{4}(s) v^{2}(s) \zeta d s \\
& \leq-2 \beta^{3} \int_{0}^{1} v^{2}(s) d s+8 \beta^{3} \int_{0}^{1} \zeta^{2}(s) v^{2}(s) d s \\
& =2 \beta^{2}(3 \beta-4) \int_{0}^{1} v^{2}(s) d s
\end{aligned}
$$

where we discarded the integral of a non-negative function and then used (49). Thus the integral on the left-hand side of (50) is negative if $\beta<4 / 3$.

Remark 2. It is easy to improve the above estimate to $3 / 2$ if, instead of discarding the integral of $\zeta^{4} v^{2}$ altogether, we deduce from (48)

$$
\zeta^{4}=\left(\frac{\beta-1}{\beta}-\frac{1}{\beta} \frac{\partial f}{\partial x}\left(x_{0}\right)\right)^{2} \geq\left(\frac{\beta-1}{\beta}\right)^{2}-\frac{2}{\beta} \frac{\partial f}{\partial x}\left(x_{0}\right) .
$$

\subsection{The $\beta=\infty$ case}

In this section all assertions are verified by explicit computation.

When we take $\beta=\infty$, instead of the ODE (44) we are left with the differential inclusion

$$
\epsilon \frac{d x}{d t} \in-x+\operatorname{Sgn}(x)+h \cos (2 \pi t),
$$

where $\operatorname{Sgn}(x)$ is the multivalued sign function,

$$
\operatorname{Sgn}(x)= \begin{cases}-1, & \text { if } x<0 \\ {[-1,1],} & \text { if } x=0 \\ 1, & \text { if } x=1 .\end{cases}
$$

Note that if a periodic solution $x(t)$ has no internal zeroes, it solves a simple linear ODE and can be computed explicitly. For example, a for $h$ sufficiently small, a positive periodic solution is given by

$$
x(t)=1+h \frac{\cos (2 \pi t)+2 \pi \epsilon \sin (2 \pi t)}{1+4 \pi^{2} \epsilon^{2}},
$$

from which we conclude that this (asymmetric) solution touches zero when

$$
h=h_{c a} \equiv \sqrt{1+4 \pi^{2} \epsilon^{2}} .
$$

For example, if $\epsilon=0.05, h_{c a}=1.048187027$ to 10 d.p. 

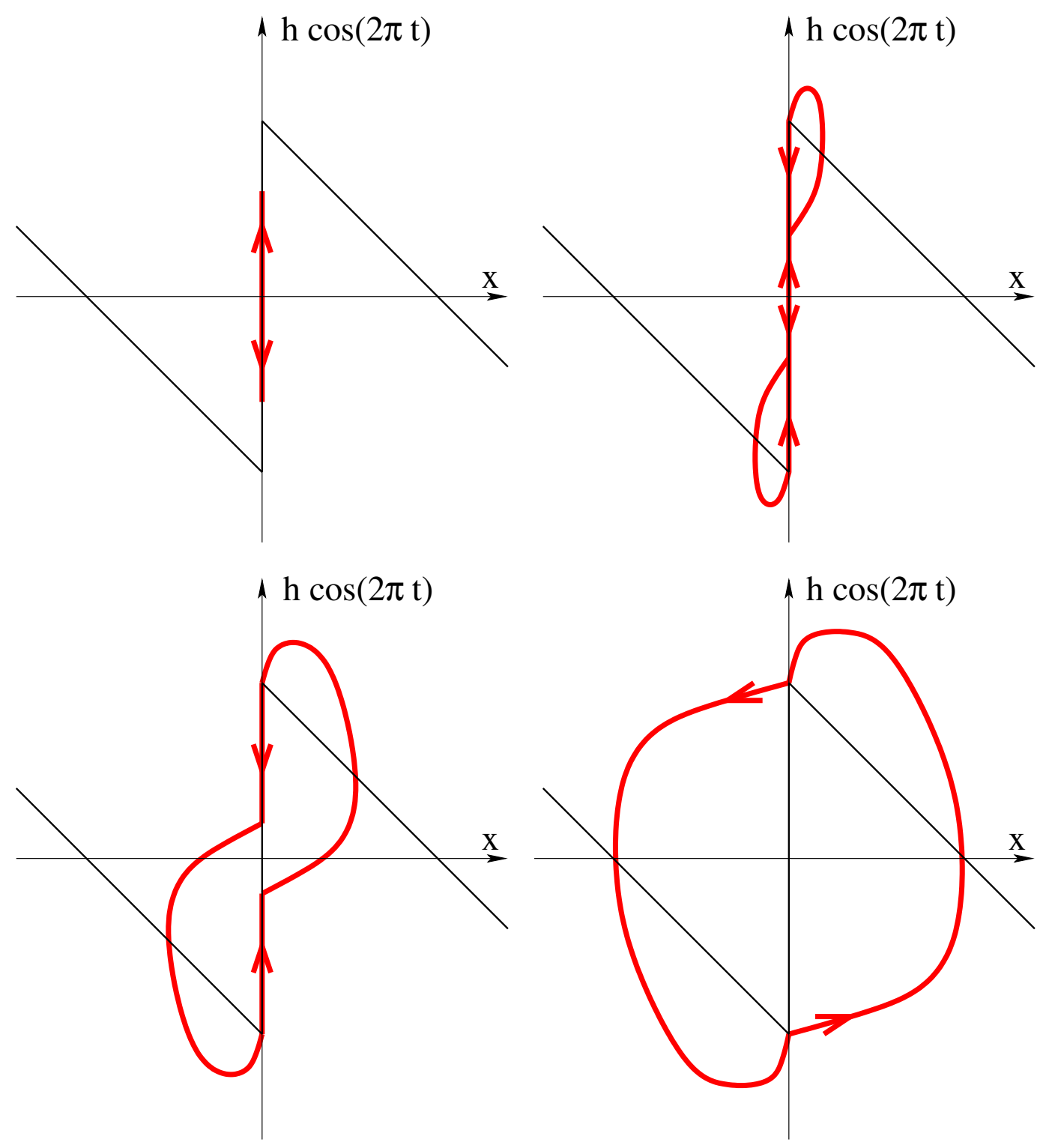

Figure 4. Evolution of the unstable zero-mean solution of inclusion (52) for $h \leq h_{c s}$. Top left: solution is identically zero when $h<1$. Top right and bottom left: emergence and growth of nontrivial parts. The solution will experience a bifurcation when the trivial parts, where $x(t)=0$ on an open interval, disappear completely. This happns at $h=h_{c s}$ (bottom right).

Next we want to understand the bifurcation 9 of the zero-mean solution, figure [4 When $h<1$, the zero-mean solution is identically zero and is unstable. After $h$ crosses 1 , the solution gets two symmetric non-trivial parts, but there are still two intervals in $t$ on which $x(t)$ is identically zero. The bifurcation happens when each of these intervals collapses to a point.

Let $h_{c s}$ be the unique value of $h$ for which there exists a periodic solution $x_{c}(t)$ of I a complete bifurcation picture and animated periodic solutions described in this section are also available at http://www.math.tamu.edu/〜berko/bistable 

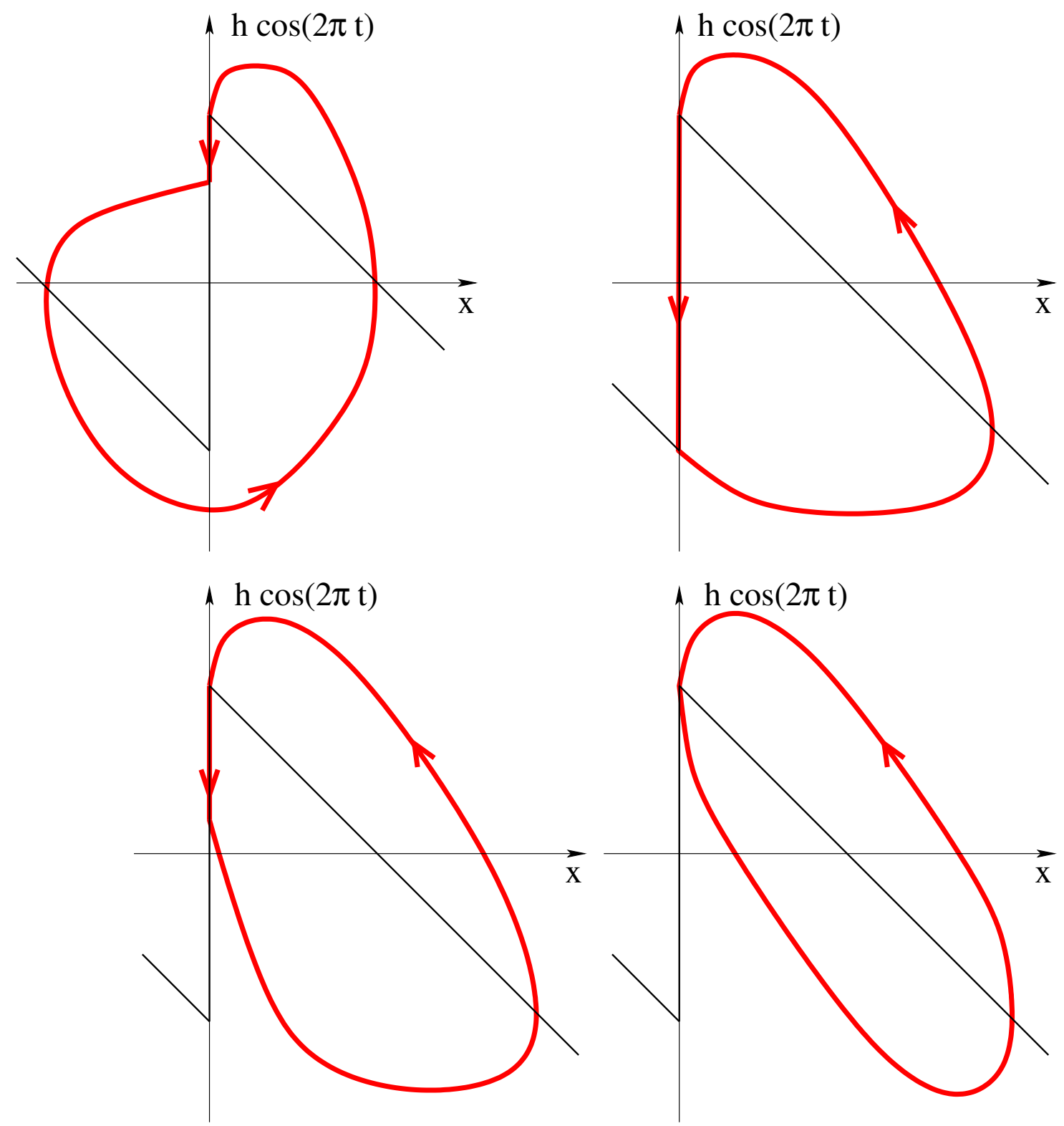

Figure 5. The evolution of the right unstable solution until its annihilation at $h=h_{c a}$.

the differential inclusion with the property that there is $t_{0}$ such that $x_{c}^{\prime}\left(t_{0}\right)=1$ and $x_{c}^{\prime}\left(t_{0}+1 / 2\right)=-1$, see figure 4 (bottom right).

An involved but elementary computation (see Appendix A) shows that this happens for

$$
h=h_{c s} \equiv \frac{\sqrt{\left(1+4 \pi^{2} \epsilon^{2}\right)\left(\pi^{2} \epsilon^{2}-2 \pi^{2} \epsilon^{2} z+\left(1+\pi^{2} \epsilon^{2}\right) z^{2}\right)}}{\pi(1+z) \epsilon},
$$

where we have put $z=\exp (-1 / 2 \epsilon)$. Expanding this expression in $z$, we obtain the following expression:

$$
h_{c s}=h_{c a}-2 h_{c a} z+O\left(z^{2}\right),
$$

from which it is clear that for small $\epsilon$ (and hence for very small $z$ ) $h_{c s}<h_{c a}$. For 


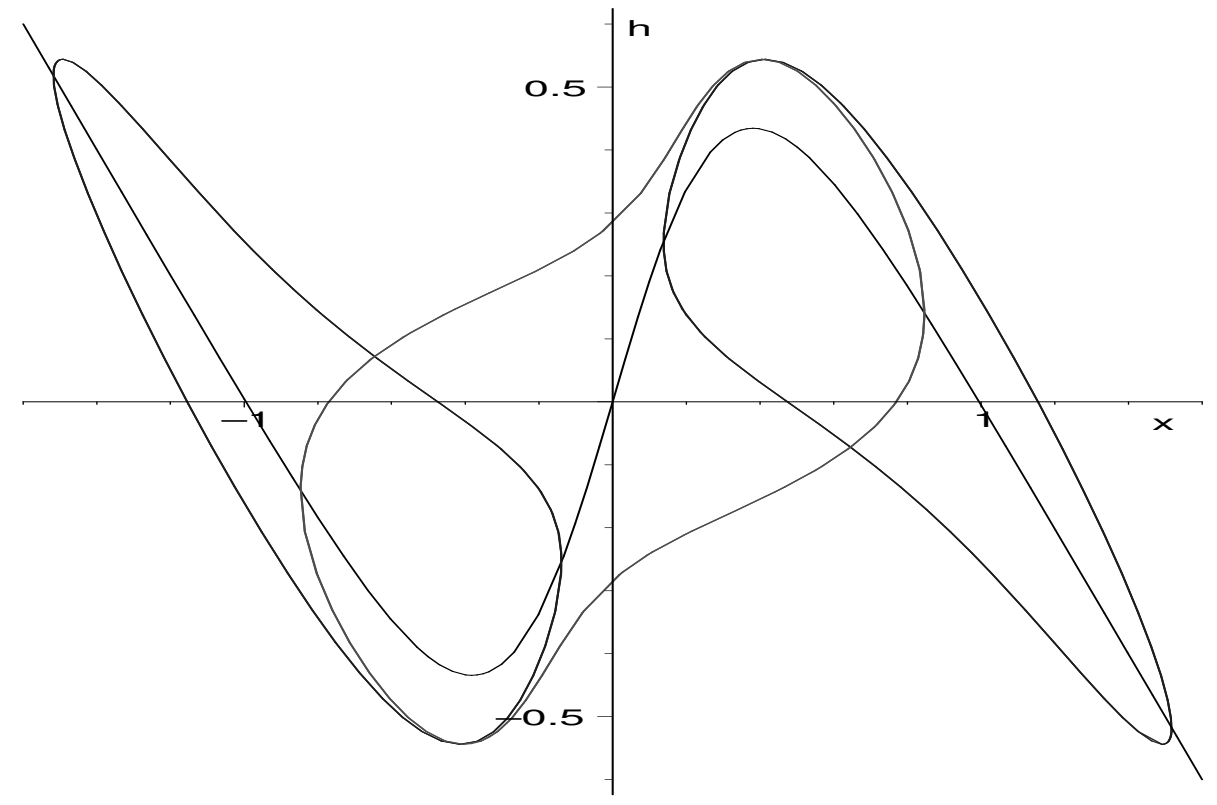

Figure 6. Three coexisting stable solutions of the Suzuki-Kubo equation for $\beta=3$, $\epsilon=0.05$ and $h=0.544$.

example, if $\epsilon=0.05, h_{c s}=1.048091900$ and therefore $h_{c a}-h_{c s}=O\left(10^{-4}\right)$.

When $h=h_{c s}$, the zero mean solution becomes stable through a super-critical pitchfork bifurcation. It emits a couple of unstable solutions which disappear in a blue-sky catastrophe, i.e. in a collision with the stable solutions (54). These unstable solutions are sketched in Figs. 5 for various values of $h, h_{c s}<h \leq h_{c a}$.

We summarise the discussion of the inclusion (52) in a theorem

Theorem 4.2. For $h$ satisfying $h_{c s}<h<h_{c a}$, the inclusion (52) has five 1-periodic solutions.

\subsection{The case of large $\beta$}

It is possible to extend the existence of 5 solutions from $\beta=\infty$ case to the case of large $\beta$ by continuity. In our numerical experiments we found that $\beta$ does not have to be very large. Figure 6 contains the plot of 3 stable solutions of equation (41) with $\beta=3, \epsilon=0.05$, $h=0.544$ and initial conditions $x(0)=-0.413600598,-0.40456237,1.491606074$.

Theorem 4.3. For any $h$ satisfying $h_{c s}<h<h_{c a}$ and sufficiently large (depending on h) $\beta$ equation (4) has at least five periodic solutions.

The proof of this theorem is based on an upper semicontinuity result for differential inclusions [2]. To state it, we need to introduce some notation. Let $B$ be the unit ball in $\mathbb{R}^{n}$ with centre at the origin. Consider a differential inclusion

$$
x^{\prime}(t) \in F(t, x(t), \lambda),
$$

$(t, x, \lambda) \in[0, T] \times \Omega \times \Lambda$, where $\Omega$ is a bounded subset of $\mathbb{R}^{n}, \Lambda \subset \mathbb{R}$ and $F$ is an upper semicontinuous set-valued map with uniformly bounded compact images. Assume 
that for all $\lambda \in \Lambda$ and any $x_{0}$ in some set $Q \in \Omega$ the solutions of the differential inclusion with $x(0)=x_{0}$ exist on the interval $[0, T]$ and remain in $\Omega$. The Poincaré map $\mathcal{P}_{\lambda}^{T}\left(x_{0}\right): Q \rightarrow \mathbb{R}^{n}$ is the set-valued map defined by

$$
\mathcal{P}_{\lambda}^{T}\left(x_{0}\right)=\left\{x(T): x^{\prime}(t) \in F(t, x(t), \lambda), x(0)=x_{0}\right\} .
$$

The following theorem is a slight variation of the continuity theorems in Section 2.2 of 2 .

Theorem 4.4 (Dependence on Parameter). Let the map $(t, x, \lambda) \rightarrow F(t, x, \lambda)$ satisfy the conditions of the previous paragraph. Then for any $\lambda_{0} \in \Lambda$ and any open set $U \subset \mathbb{R}^{2 n}, 0 \in U$, there is a $\delta>0$ such that $\left|\lambda-\lambda_{0}\right|<\delta$ implies

$$
\operatorname{graph}\left(\mathcal{P}_{\lambda}^{T}\right) \subset \operatorname{graph}\left(\mathcal{P}_{\lambda_{0}}^{T}\right)+U \text {. }
$$

Proof of Theorem 4.3. Clearly, $F(t, x, \beta)$ defined by the right-hand side (4) viewed as a differential inclusion satisfies the conditions of Theorem 4.4 since all solutions of this inclusion are globally defined and remain bounded.

Fix $h \in\left(h_{c s}, h_{c a}\right)$. By theorem 4.4, for sufficiently large $\beta$ the graph of the Poincaré map $\mathcal{P}_{\beta}^{1}$ of the Suzuki-Kubo equation (4) is contained in a neighbourhood of the graph of $\mathcal{P}_{\infty}$. By Theorem 4.2, the graph has five intersections with the diagonal. Therefore, for sufficiently small neighbourhood, i.e. for sufficiently large $\beta$, we get at least five 1-periodic solutions of (44).

\section{Conclusions}

In this paper we have resolved a minor controversy in the physical literature. However, some major questions still remain. One we can formulate as a conjecture:

Conjecture 1. An ODE of the form of (6) with $f(y)$ bistable has at most five 1-periodic solutions.

The other fundamental question is to formulate necessary conditions on $f(y)$ for (6) to have a specified maximum number of solutions. We end with two remarks.

(i) Consider (6) in which the nonlinearity $f(y)$ is piecewise linear,

$$
f(y)= \begin{cases}-1-y & \text { if } y<-1 / 2 \\ y & \text { if } y \in[-1 / 2,1 / 2] \\ 1-y & \text { if } y>1 / 2 .\end{cases}
$$

Then it can be shown, by explicitly constructing the stable periodic solutions, that there is a range of $h$ for which (6) with such a nonlinearity will have five 1-periodic solutions 4. This perhaps indicates that there may be more than one mechanism for the appearance and disappearance of solutions.

(ii) A possible way to attack the problem of necessary conditions is to consider a homotopy of (2) into (11), i.e.

$$
x^{\prime}=(1-2 \alpha) x+(1-\alpha) \tanh (\beta x)-\alpha x^{3}+h \cos (2 \pi t),
$$


and see, at least numerically, for which value $\alpha$ one no longer has a subcritical bifurcation from the symmetric solution.

\section{Acknowledgement}

The authors gratefully acknowledge fruitful discussions with J. Byatt-Smith, B. Duffy and J. Carr.

\section{Appendix A. Calculating $h_{c s}$}

The definition of $h_{c s}$ is the value of $h$ for which there exists a periodic solution $x_{c}(t)$ of equation

$$
\epsilon \frac{d x}{d t}=1-x+h \cos (2 \pi t)
$$

with the property that it connects the points $(0,-1)$ and $(0,1)$ (see diagram). In other words, the following equations are satisfied

$$
\begin{array}{ll}
h \cos \left(2 \pi t_{0}\right) & =1, \\
x_{c}\left(t_{0}\right) & =0, \\
x_{c}\left(t_{0}+1 / 2\right) & =0
\end{array}
$$

The general solution of equation (A.1) is

$$
x(t)=C_{1} e^{-t / \epsilon}+\frac{1+4 \pi^{2} \epsilon^{2}+h \cos (2 \pi t)+2 h \pi \epsilon \sin (2 \pi t)}{1+4 \pi^{2} \epsilon^{2}}
$$

From (A.2) we get $h \sin \left(2 \pi t_{0}\right)=\sqrt{h^{2}-1}$. Equations (A.3)-(A.4) then read

$$
\begin{aligned}
& C_{1} e^{-t_{0} / \epsilon}+\frac{2+4 \pi^{2} \epsilon^{2}+2 \pi \epsilon \sqrt{h^{2}-1}}{1+4 \pi^{2} \epsilon^{2}}=0 \\
& C_{1} e^{-t_{0} / \epsilon} e^{-1 / 2 \epsilon}+\frac{4 \pi^{2} \epsilon^{2}-2 \pi \epsilon \sqrt{h^{2}-1}}{1+4 \pi^{2} \epsilon^{2}}=0
\end{aligned}
$$

We eliminate $C_{1} e^{-t / \epsilon}$ to get

$$
e^{-1 / 2 \epsilon}\left(2+4 \pi^{2} \epsilon^{2}+2 \pi \epsilon \sqrt{h^{2}-1}\right)-4 \pi^{2} \epsilon^{2}+2 \pi \epsilon \sqrt{h^{2}-1}=0
$$

Denoting $e^{-1 / 2 \epsilon}=z$, we finally obtain

$$
h_{c s}=\frac{\sqrt{\left(1+4 \pi^{2} \epsilon^{2}\right)\left(\pi^{2} \epsilon^{2}-2 \pi^{2} \epsilon^{2} z+\left(1+\pi^{2} \epsilon^{2}\right) z^{2}\right)}}{\pi \epsilon(1+z)}
$$

\section{References}

[1] M. Acharyya and B. K. Chakrabarti, Magnetic hysteresis loops as Lissajous plots of relaxationally delayed response to periodic field variation, Physica A 202 (1994), 467-481.

[2] J.-P. Aubin and A. Cellina, Differential Inclusions, Springer-Verlag, Berlin 1984.

[3] N. Berglund, Adiabatic Dynamical Systems and Hysteresis, Ph. D. thesis, Institut de Physique Théorique, EPFL, Lausanne 1998. 
[4] G. Berkolaiko, Lecture at University of Strathclyde Analysis Seminar, 1997.

[5] J. Byatt-Smith, Unpublished notes, 2002.

[6] M. Golubitsky and D. G. Schaeffer, Singularities and Groups in Bifurcation Theory, Vol.I, Springer-Verlag, New York 1985.

[7] A. Hohl et al., Scaling laws for dynamical hysteresis in a multidimensional laser system, Phys. Rev. Lett. 74 (1995), 2220-2223.

[8] P. Jung, G. Gray, R. Roy, and P. Mandel, Scaling law for dynamical hysteresis, Phys. Rev. Lett. 65 (1991), 1873-1876.

[9] G. Korniss, P. A. Rikvold and M. A. Novotny, Absence of first-order transition and tricritical point in the dynamic phase diagram of a spatially extended bistable system in an oscillating field, Phys. Rev. E 66 (2002), 056127.

[10] V. A. Pliss, Nonlocal Problems of the Theory of Oscillations, Academic Press, New York 1966.

[11] M. Rao, H. R. Krishnamurthy, and R. Pandit, Magnetic hysteresis in two model spin systems, Phys. Rev. B 42 (1990), 856-884.

[12] M. Suzuki and R. Kubo, Dynamics of the Ising model near the critical point. I, J. Phys. Soc. Japan 24 (1968), 51-60.

[13] T. Tomé and M. J. de Oliveira, Dynamic phase transition in the kinetic Ising model under a time-dependent oscillating field, Phys. Rev. A 41 (1990), 4251-4254.

[14] M. F. Zimmer, Ising-model in an oscillating magnetic field: mean-field theory, Phys. Rev. E 47 (1993), 3950-3955. 